\title{
Expression of miR-28-3p in patients with Alzheimer's disease before and after treatment and its clinical value
}

\author{
XIAOHUA ZHAO $^{1 *}$, SHAN WANG $^{1 *}$ and WENBAO SUN ${ }^{2}$ \\ ${ }^{1}$ Department of Neurology, The People's Hospital of Shouguang; ${ }^{2}$ Department of General Surgery, \\ Shouguang Hospital of TCM, Weifang, Shandong 262700, P.R. China
}

Received October 2, 2019; Accepted May 4, 2020

DOI: $10.3892 / \mathrm{etm} .2020 .8920$

\begin{abstract}
Expression of miR-28-3p in patients with Alzheimer's disease (AD) before and after treatment and clinical value of miR-28-3p were determined. There were three groups: $68 \mathrm{AD}$ patients treated with donepezil combined with basic therapy in The People's Hospital of Shouguang collected as an AD group, 70 patients with mild cognitive impairment (MCI) as an MCI group, and 75 healthy people as a normal group. Serum miR-28-3p was detected by qRT-PCR. The Montreal cognitive assessment scale (MoCA), mini mental state examination scale (MMSE), activities of daily living scale (ADL) and homocysteine (Hcy) were adopted to assess patients before and after treatment. miR-28-3p in normal group was significantly lower than that in other two groups, and miR-28-3p in MCI group was significantly lower than that in AD group $(\mathrm{P}<0.001)$. miR-28-3p correlated with the course and severity of patients. miR-28-3p in AD group after treatment was significantly lower than that before treatment $(\mathrm{P}<0.001)$. ADL and Hcy of AD patients after treatment were significantly lower than before treatment $(\mathrm{P}<0.05)$, and MMSE and MoCA after treatment were significantly higher than before treatment $(\mathrm{P}<0.05)$. Before and after treatment, miR-28-3p was significantly positively correlated with ADL score and Hcy level, but negatively correlated with MMSE score and MoCA score. Analysis of the working characteristic curve of the patients indicated that miR-28-3p can be used for diagnosis of AD patients. Donepezil therapy may reduce miR-28-3p level to alleviate the symptoms of AD patients, and miR-28-3p level can be used as an early diagnosis and prognosis evaluation of $\mathrm{AD}$ patients.
\end{abstract}

Correspondence to: Dr Wenbao Sun, Department of General Surgery, Shouguang Hospital of TCM, 3353 Shengcheng Street, Weifang, Shandong 262700, P.R. China

E-mail: s3k2rs@163.com

*Contributed equally

Key words: miR-28-3p, Alzheimer's disease, donepezil

\section{Introduction}

Alzheimer's disease (AD) is a common neurodegenerative disease in the elderly, mainly manifested as cognitive dysfunction, memory decline, social disorders, and behavioral abnormalities $(1,2)$. AD patients account for more than $80 \%$ of dementia cases among people aged over 65 years in the world (3). According to previous reports, it was estimated that 47.5 million people worldwide would suffer from dementia in 2015, and the incidence rate of AD shows an increasing trend with changes in lifestyle (4,5). Previous studies showed that the loss of cholinergic nerve cells in basal forebrain and the increase of acetylcholinesterase (ACh E) activity in AD patients lead to the decrease of neurotransmitter acetylcholine (ACh). Therefore, the therapeutic purpose was mainly achieved by inhibiting ACh E activity (6). Donepezil, one of acetylcholinesterase inhibitors, is a commonly used drug for the treatment of AD and has a good effect, which can significantly improve the cognitive function of AD patients (7).

At present, many researchers are looking for biomarkers that can detect the disease and monitor the course of the disease, especially before symptoms appear or in early stages $(8,9)$. In recent years, the exploration of peripheral blood microRNA (miRNA) has become a research hot-spot. Some studies have reported that there are some changes in miRNA levels in postmortem brain studies and changes in miRNA in whole blood, plasma or serum. Therefore, the detection of the changes of miRNA in AD has high clinical value for the early diagnosis and curative effect evaluation of the disease (10-13). miRNA belongs to an endogenous non-coding protein RNA gene (with the length of about 18-24 nucleotides), which can play an important role in a variety of diseases. For example, it mediates the regulation of protein production by interacting with target messenger RNA (mRNA) (14). miR-28-3p is a miRNA that targets a variety of cancer-related genes and can participate in epithelial-mesenchymal transition (EMT), cell proliferation, invasion and migration $(15,16)$. Moreover, studies have showed that miR-28-3p was significantly downregulated in nasopharyngeal carcinoma tissues and could accelerate the invasion and migration of nasopharyngeal carcinoma cells (16). Hong et al (17) showed that miR-28-3p was significantly upregulated in AD APP/PS1 transgenic mouse model. Paltsev et al (18) reported that miR-132 was involved 
in the pathogenesis of senile dementia by detecting the serum miR-132 level of dementia patients, and it could be used as a detection index for early diagnosis and treatment of senile dementia. However, there is no research report on whether miR-28-3p plays the same role in AD patients.

In this study, miR-28-3p in serum of AD patients before and after treatment was determined, and its correlation with clinical indicators was analyzed, to provide certain reference for early diagnosis and treatment of AD.

\section{Patients and methods}

Clinical general data. Altogether 68 AD patients admitted to The People's Hospital of Shouguang (Weifang, China) were collected as the AD group, including 31 males and 37 females, with an average age of $70.12 \pm 2.09$ years, with the MoCA score of $14.67 \pm 2.01$ and MMSE score of $15.48 \pm 1.68$. Further 70 patients with early cognitive impairment were treated as the MCI group, including 32 males and 38 females, with an average age of $69.68 \pm 2.11$ years, with the MoCA score of $20.76 \pm 1.69$ and MMSE score of $22.67 \pm 0.73$. Additionally 75 healthy people who underwent physical examination in The People's Hospital of Shouguang during the same period were selected as the normal group, including 34 males and 41 females, with an average age of $69.47 \pm 1.98$ years, with the MoCA score of $27.82 \pm 1.42$ and MMSE score of $28.03 \pm 1.52$. This study was approved by the Ethics Committee of The People's Hospital of Shouguang. The patients and their families were informed in advance and signed a complete informed consent form.

Inclusion criteria: Patients with good compliance and complete clinical data; the educational level of the patients was primary school or above; all patients received routine examinations on urine routine, blood routine, electrocardiogram, liver and kidney functions after admission; patients were accompanied by their family when they were admitted to hospital.

Exclusion criteria: Patients with previous history of mental illness, liver dysfunction, severe organ lesions, craniocerebral trauma, and autoimmune system defects; patients who had a history of drug dependence and had taken antidepressants; patients who had cognitive impairments.

Treatment methods. Patients with AD were treated with donepezil as basic therapy. After admission, patients were given drugs to improve cerebral blood circulation and promote brain cell metabolism. According to the specific conditions of the patients, symptomatic treatment was given, and the rest condition and diet of the patients were adjusted. Patients should take moderate rehabilitation exercise and get adequate sleep. At the same time, donepezil hydrochloride tablets (Chinese Eisai Pharmaceutical Co., Ltd., SFDA approval no. H20050978) were given on this basis, once a day and $5 \mathrm{mg}$ for the first time. After one month of continuous treatment, the stable blood drug concentration and clinical response to the drug were evaluated. According to the specific situation, the dosage was increased to $10 \mathrm{mg}$ once a day for one year.

Detection index. A total of $4 \mathrm{ml}$ of fasting elbow venous blood were collected from the three groups of patients after admission and from AD patients after discharge. After coagulation for
$60 \mathrm{~min}\left(20-25^{\circ} \mathrm{C}\right)$, each sample was centrifuged at $1,006.2 \mathrm{x} \mathrm{g}$ for $10 \mathrm{~min}$ to collect the upper serum. The centrifugation radius was $10 \mathrm{~cm}$ and the centrifugation temperature was $4^{\circ} \mathrm{C}$. The collected serum was placed in a refrigerator at $-80^{\circ} \mathrm{C}$ for testing. The level of homocysteine (Hcy) was detected by cyclic enzyme method (19). The level of miR-28-3p was detected by real-time fluorescence quantitative PCR (qRT-PCR). Total RNA in serum was extracted according to the instructions of TRIzol kit (Shanghai Sangon Biotech). The template RNA was digested and treated with DNase I (Shanghai Sangon Biotech) to eliminate DNA contamination. The ultraviolet spectrophotometer (Beijing Up General Technology Co., Ltd.) was used to measure the purity and concentration, and OD 260/280 value more than 1.8 was considered usable. The RNA sample was then reverse transcribed into cDNA, and the operation was strictly carried out in accordance with the instructions of cDNA reverse transcription kit (Takara Bio). qRT-PCR was used for detection. The qRT-PCR reaction was carried out on ABI 7500 system (Applied Biosystems) using SYBR-Green PCR Master Mix (Thermo Fisher Scientific). PCR reaction conditions were as follows: pre-denaturation at $95^{\circ} \mathrm{C}$ for $10 \mathrm{~min}$, denaturation at $95^{\circ} \mathrm{C}$ for $10 \mathrm{sec}$, and annealing and extension at $60^{\circ} \mathrm{C}$ for $60 \mathrm{sec}$, for a total of 40 cycles. Primers for this experiment were designed by Premier 5.0 (Premier) and generated by Tianjin Saierbio Co., Ltd. U6 was used as internal reference. The specific primer sequences are shown in Table I. The above system configuration was strictly in accordance with the instructions. The level of miR-28-3p was calculated by $2^{-\Delta \mathrm{Ct}}$.

Observation indicators. i) The miR-28-3p levels of patients in MCI group, normal group and AD group were compared. ii) The relationship between miR-28-3p level and clinical data in AD patients was compared. iii) Montreal cognitive assessment scale (MoCA) (20) and mini mental state examination scale (MMSE) (21) were used to evaluate the cognitive function and mental state of MCI group, normal group and AD group before treatment and 3 months after treatment, with a total score of 30 points. The high score was closely related to the good cognitive function. The levels of activity of daily life (ADL) and Hcy were observed before treatment and 3 months after treatment in AD group. ADL was used to evaluate the patients' activity of life. The high score was closely related to poor activity of life (22). iv) The correlation among serum miR-28-3p level, score, and Hcy level in AD patients was analyzed. v) The clinical value of miR-28-3p in diagnosing AD patients was explored.

Statistical analysis. SPSS 20.0 (IBM Corp.) was used for statistical analysis. GraphPad Prism 7 (GraphPad Software Co., Ltd.) was used to illustrate the collected data. The count data was expressed as n (\%), and chi-square test was used for inter-group comparison. The measurement data was expressed as mean \pm standard deviation (mean \pm SD). The t-test was used for comparison between the two groups, one-way ANOVA was used for comparison among multiple groups, which was expressed as F. LSD-t-test was used for post-event analysis, Pearson's analysis was used for bivariate correlation analysis, and receiver operating characteristic curve (ROC) was used for diagnosis of $\mathrm{AD}$ patients. $\mathrm{P}<0.05$, was considered a statistically significant difference. 
Table I. Primer sequences.

\begin{tabular}{lcc}
\hline Gene & Upstream & Downstream \\
\hline miR-28-3p & CGCGCACTAGATTGTGAGCT & AGTGCAGGGTCCGAGGTATT \\
U6 & CGACAAGACGATCCGGGTAAA & GGTTGAGGAGTGGGTCGAAG \\
\hline
\end{tabular}

Table II. Comparison of general clinical data of three groups [mean $\pm \mathrm{SD}, \mathrm{n}(\%)]$.

\begin{tabular}{|c|c|c|c|c|c|}
\hline Clinical data & $\begin{array}{l}\text { AD group } \\
(n=68)\end{array}$ & $\begin{array}{l}\text { MCI group } \\
\quad(n=70)\end{array}$ & $\begin{array}{l}\text { Normal group } \\
\quad(n=75)\end{array}$ & $\mathrm{F} / \chi^{2}$ value & P-value \\
\hline Sex & & & & 0.002 & 0.999 \\
\hline Male & 31 (45.59) & $32(45.71)$ & $34(45.33)$ & & \\
\hline Female & 37 (54.41) & $38(54.29)$ & $41(54.67)$ & & \\
\hline Average age (years) & $70.12 \pm 2.09$ & $69.68 \pm 2.11$ & $69.47 \pm 1.98$ & 1.832 & 0.163 \\
\hline Smoking & & & & 0.273 & 0.873 \\
\hline Yes & $24(35.29)$ & $22(31.43)$ & $24(32.00)$ & & \\
\hline No & $44(64.71)$ & $48(68.57)$ & $51(68.00)$ & & \\
\hline Drinking & & & & 3.496 & 0.174 \\
\hline Yes & 47 (69.12) & 48 (68.57) & $42(56.00)$ & & \\
\hline No & $21(30.88)$ & $22(31.43)$ & $33(44.00)$ & & \\
\hline Body mass index $\left(\mathrm{kg} / \mathrm{m}^{2}\right)$ & $23.49 \pm 3.27$ & $24.07 \pm 3.31$ & $23.95 \pm 4.06$ & 0.505 & 0.604 \\
\hline \multicolumn{6}{|l|}{ Complication } \\
\hline Diabetes & 27 (39.71) & $25(35.71)$ & $22(29.33)$ & 1.736 & 0.420 \\
\hline Hypertension & $36(52.94)$ & $33(47.14)$ & $37(49.33)$ & 0.473 & 0.790 \\
\hline $\mathrm{TG}(\mathrm{mmol} / \mathrm{l})$ & $1.68 \pm 0.72$ & $1.61 \pm 0.67$ & $1.57 \pm 0.77$ & 0.420 & 0.657 \\
\hline $\mathrm{TC}(\mathrm{mmol} / \mathrm{l})$ & $5.29 \pm 1.12$ & $5.15 \pm 1.04$ & $5.10 \pm 0.99$ & 0.620 & 0.5391 \\
\hline HDL-C (mmol/l) & $1.30 \pm 0.56$ & $1.53 \pm 0.62^{\mathrm{a}}$ & $1.91 \pm 0.87^{\mathrm{a}, \mathrm{b}}$ & 13.460 & $<0.001$ \\
\hline LDL-C (mmol/l) & $2.98 \pm 1.13$ & $2.82 \pm 1.07$ & $2.54 \pm 1.10^{\mathrm{a}}$ & 2.951 & 0.055 \\
\hline MoCA score & $14.67 \pm 2.01$ & $20.76 \pm 1.69^{\mathrm{a}}$ & $27.82 \pm 1.42^{\mathrm{a}, \mathrm{b}}$ & 1.055 & $<0.001$ \\
\hline MMSE score & $15.48 \pm 1.68$ & $22.67 \pm 0.73^{\mathrm{a}}$ & $28.03 \pm 1.52^{\mathrm{a}, \mathrm{b}}$ & 1.491 & $<0.001$ \\
\hline
\end{tabular}

${ }^{\text {aP }}<0.05$, compared with $\mathrm{AD}$ group; ${ }^{\mathrm{b}} \mathrm{P}<0.05$, compared with $\mathrm{MCI}$ group. AD, Alzheimer's disease; MCI, mild cognitive impairment; TG, triglyceride; TC, total cholesterol; HDL-C, high density lipoprotein cholesterol; LDL-C, low density lipoprotein cholesterol; MoCA, Montreal cognitive assessment scale; MMSE, mini mental state examination scale.

\section{Results}

Comparison of general clinical data of three groups. There was no significant difference in sex, average age, smoking and drinking, body mass index, complications, triglyceride (TG) or total cholesterol (TC) among AD, MCI and normal groups ( $\mathrm{P}>0.05)$. The high density lipoprotein cholesterol (HDL-C), MoCA score and MMSE score of AD group were significantly lower than those of the other two groups, while the low density lipoprotein cholesterol (LDL-C) of AD group was higher than that of the normal group $(\mathrm{P}<0.05)$ (Table II).

Comparison of miR-28-3p levels in three groups. Comparing the serum miR-28-3p levels of AD group, MCI group and normal group, as shown in Fig. 1, it was clear that the miR-28-3p content of normal group was significantly lower than that of AD group and MCI group $(\mathrm{P}<0.05)$, while the serum miR-28-3p level of MCI group was significantly lower than that of $\mathrm{AD}$ group patients $(\mathrm{P}<0.001)$.

Relationship between serum miR-28-3p level and clinical data in AD patients. The clinical data of AD patients were collected as shown in Table III. The miR-28-3p level had no significant correlation with sex, age, smoking or drinking ( $P>0.05$ ), however, miR-28-3p level had a significant correlation with disease course and severity $(\mathrm{P}<0.05)$.

Comparison of serum miR-28-3p levels of AD patients before and after treatment. Comparison of the serum miR-28-3p level of $\mathrm{AD}$ patients before and after treatment in Fig. 2, indicated that the serum miR-28-3p level of AD patients before treatment was $3.07 \pm 0.71$, the serum miR-28-3p level after 
Table III. Relationship between serum miR-28-3p level and clinical characteristics in AD patients (mean \pm SD).

\begin{tabular}{|c|c|c|c|c|}
\hline Clinical characteristics & $\mathrm{n}$ & miR-28-3p & $\mathrm{F} / \mathrm{t}$ value & P-value \\
\hline Sex & & & 0.487 & 0.628 \\
\hline Male & 37 & $3.11 \pm 0.65$ & & \\
\hline Female & 31 & $3.04 \pm 0.51$ & & \\
\hline Age & & & 0.785 & 0.435 \\
\hline$<65$ years & 20 & $3.02 \pm 0.45$ & & \\
\hline$\geq 65$ years & 48 & $3.12 \pm 0.49$ & & \\
\hline Course of disease & & & 3.058 & 0.003 \\
\hline$<2$ years & 42 & $2.89 \pm 0.44$ & & \\
\hline$\geq 2$ years & 26 & $3.25 \pm 0.49$ & & \\
\hline Smoking & & & 0.405 & 0.687 \\
\hline Yes & 24 & $3.10 \pm 0.46$ & & \\
\hline No & 44 & $3.05 \pm 0.50$ & & \\
\hline Drinking & & & 1.101 & 0.275 \\
\hline Yes & 47 & $3.15 \pm 0.51$ & & \\
\hline No & 21 & $3.01 \pm 0.42$ & & \\
\hline Severity & & & 10.280 & $<0.001$ \\
\hline Mild & 22 & $2.75 \pm 0.37$ & & \\
\hline Medium & 31 & $3.09 \pm 0.42$ & & \\
\hline Severe & 15 & $3.37 \pm 0.47$ & & \\
\hline
\end{tabular}

$\mathrm{AD}$, Alzheimer's disease.

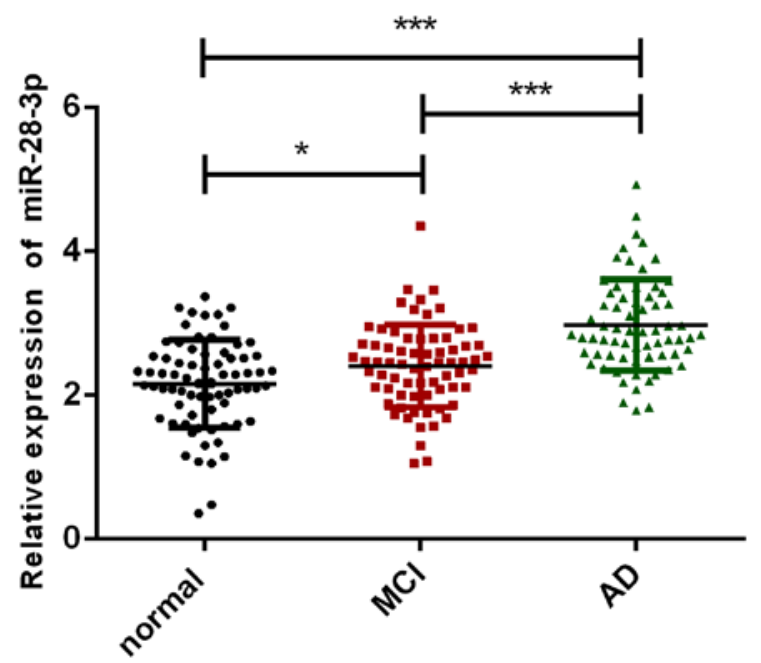

Figure 1. Comparison of serum miR-28-3p levels among the three groups. The miR-28-3p level in normal group was significantly lower than that in AD group and $\mathrm{MCI}$ group $(\mathrm{P}<0.05)$, while the serum miR-28-3p level in MCI group was significantly lower than that in $\mathrm{AD}$ group $(\mathrm{P}<0.001)$. "P<0.05 indicates the comparison between the two groups; ${ }^{* * *} \mathrm{P}<0.001$ indicates the comparison between the two groups. AD, Alzheimer's disease; $\mathrm{MCI}$, mild cognitive impairment.

treatment was $2.55 \pm 0.61$, and the serum miR-28-3p level after treatment was significantly lower than that before treatment $(\mathrm{P}<0.001)$.

Changes of score and Hcy level of AD patients before and after treatment. ADL score, MMSE score and Hcy level of AD after treatment were compared. As shown in Table IV, ADL score

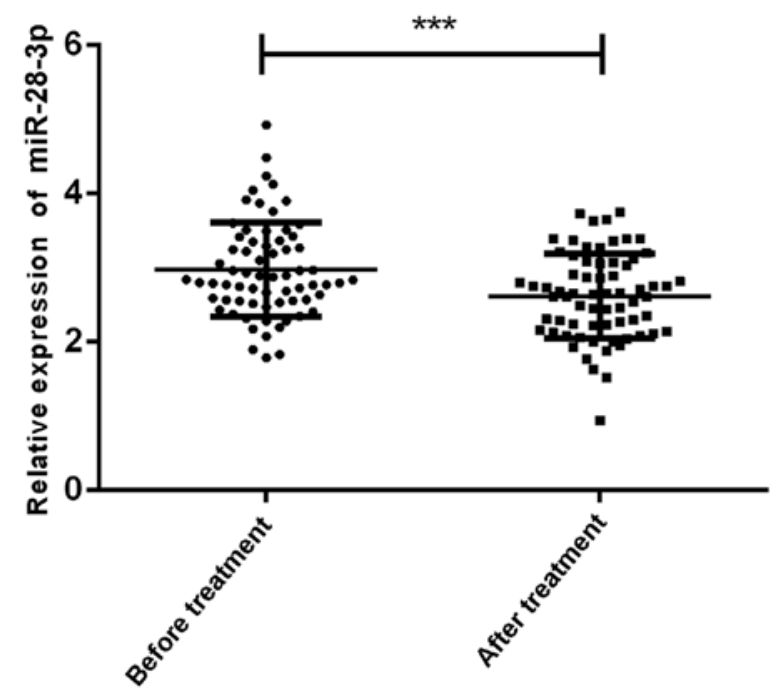

Figure 2. Serum miR-28-3p level of AD patients before and after treatment. The level of serum miR-28-3p in AD patients after treatment was significantly lower than that before treatment $(\mathrm{P}<0.001) .{ }^{* * *} \mathrm{P}<0.001$ indicates the comparison between the two groups. AD, Alzheimer's disease.

and Hcy level of AD patients after treatment were significantly lower than those before treatment $(\mathrm{P}<0.05)$. MMSE score and MoCA score after treatment were significantly higher than those before treatment $(\mathrm{P}<0.05)$.

Correlation of serum miR-28-3p level with score and Hcy level and clinical indicators of AD patients before treatment. 
Table IV. Comparison of clinical indexes of AD patients before and after treatment (mean \pm SD).

\begin{tabular}{lcccc}
\hline Treatment stage & ADL score & MMSE score & MoCA score & Hcy $(\mu \mathrm{mol} / \mathrm{l})$ \\
\hline Before treatment & $47.76 \pm 5.13$ & $15.48 \pm 1.68$ & $14.67 \pm 2.01$ & $19.21 \pm 7.68$ \\
After treatment & $43.23 \pm 4.58$ & $20.76 \pm 1.87$ & $19.89 \pm 1.88$ & $11.52 \pm 8.02$ \\
t value & 5.432 & 17.320 & 15.640 & 5.711 \\
P-value & $<0.001$ & $<0.001$ & $<0.001$ & $<0.001$ \\
\hline
\end{tabular}

AD, Alzheimer's disease; MoCA, Montreal cognitive assessment scale; MMSE, mini mental state examination scale; ADL, daily living scale; Hcy, homocysteine.
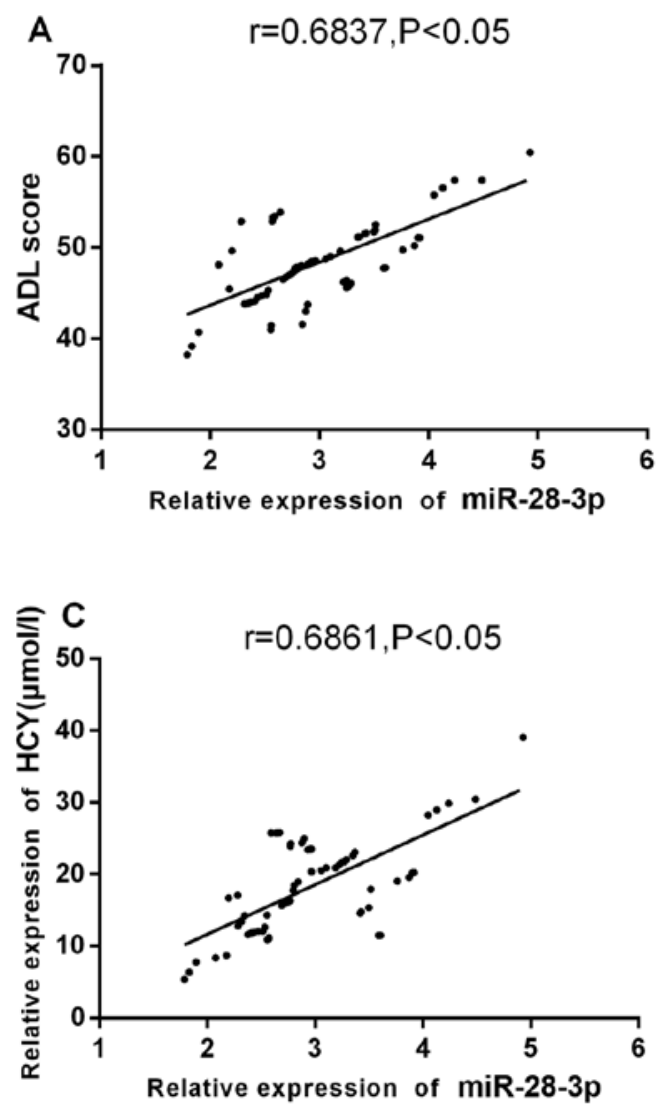
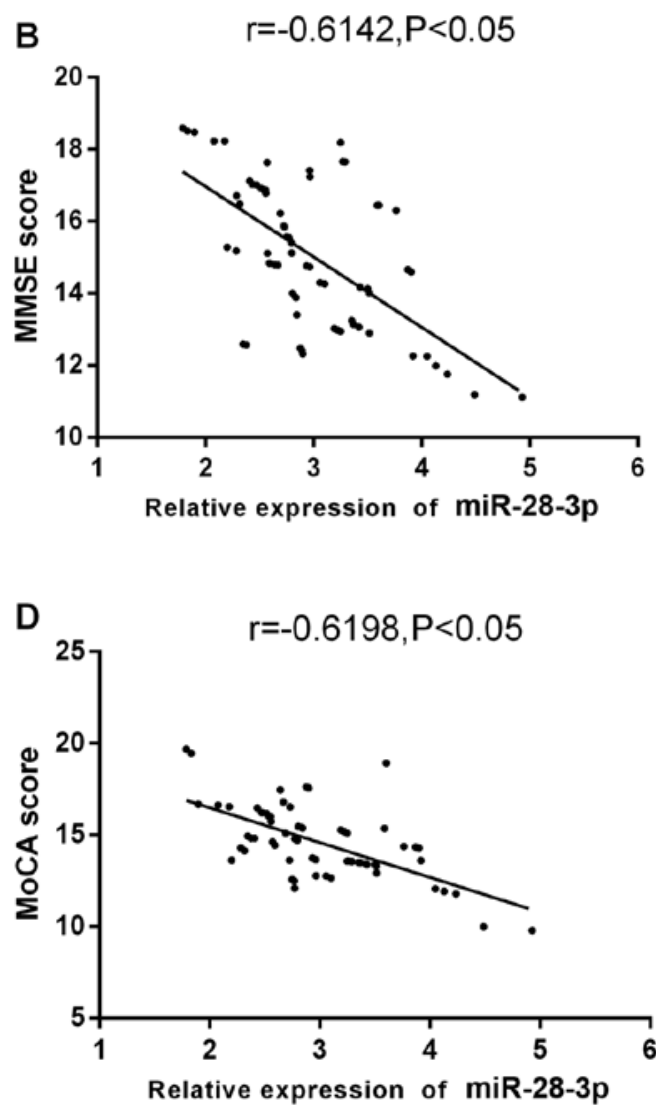

Figure 3. Correlation between serum miR-28-3p level and clinical indexes in AD patients before treatment. Pearson's analysis showing that, (A) The serum miR-28-3p level of AD patients before treatment was significantly positively correlated with ADL score $(r=0.6837, \mathrm{P}<0.05)$; $(\mathrm{B})$ the serum miR-28-3p level of AD patients before treatment was significantly negatively correlated with MMSE score $(r=-0.6142, P<0.05)$; (C) before treatment, the serum miR-28-3p level of AD patients was significantly positively correlated with Hcy level ( $\mathrm{r}=0.6861, \mathrm{P}<0.05)$; (D) miR-28-3p level was significantly negatively correlated with MoCA score $(r=-0.6198, P<0.05)$. AD, Alzheimer's disease; ADL, daily living scale; MoCA, Montreal cognitive assessment scale; Hcy, homocysteine; MMSE, mini mental state examination scale.

Pearson's analysis showed that serum miR-28-3p level was significantly positively correlated with ADL score and Hcy level before treatment $(\mathrm{r}=0.6837,0.6861, \mathrm{P}<0.05)$, and miR-28-3p level was significantly negatively correlated with MMSE score and MoCA score ( $\mathrm{r}=-0.6142,-0.6198$, $\mathrm{P}<0.05$ ) (Fig. 3).

Correlation of serum miR-28-3p level with score and Hcy correlation between serum miR-28-3p level and clinical indicators in AD patients after treatment. Pearson's analysis showed that serum miR-28-3p level was significantly positively correlated with ADL score and Hcy level after treatment $(r=0.6464,0.6824, \mathrm{P}<0.05)$, and miR-28-3p level was significantly negatively correlated with MMSE score and MoCA score ( $\mathrm{r}=-0.6598,-0.6524, \mathrm{P}<0.05)$ (Fig. 4).

Value of miR-28-3P in diagnosis of AD patients. AD group and normal group were selected to observe the diagnostic value of miR-28-3p, as shown in Fig. 5. The sensitivity of miR-28-3p for distinguishing AD patients from normal subjects was $79.41 \%$, the specificity was $74.67 \%$, the AUC was 0.8306 (0.7649-0.8963), and the cut-off value was 2.52. Patients in AD group and MCI group were selected, as shown in Fig. 6. As shown miR-28-3p had sensitivity of $64.71 \%$, specificity of $75.71 \%$, AUC of 0.7506 (0.6704-0.8309), and the 

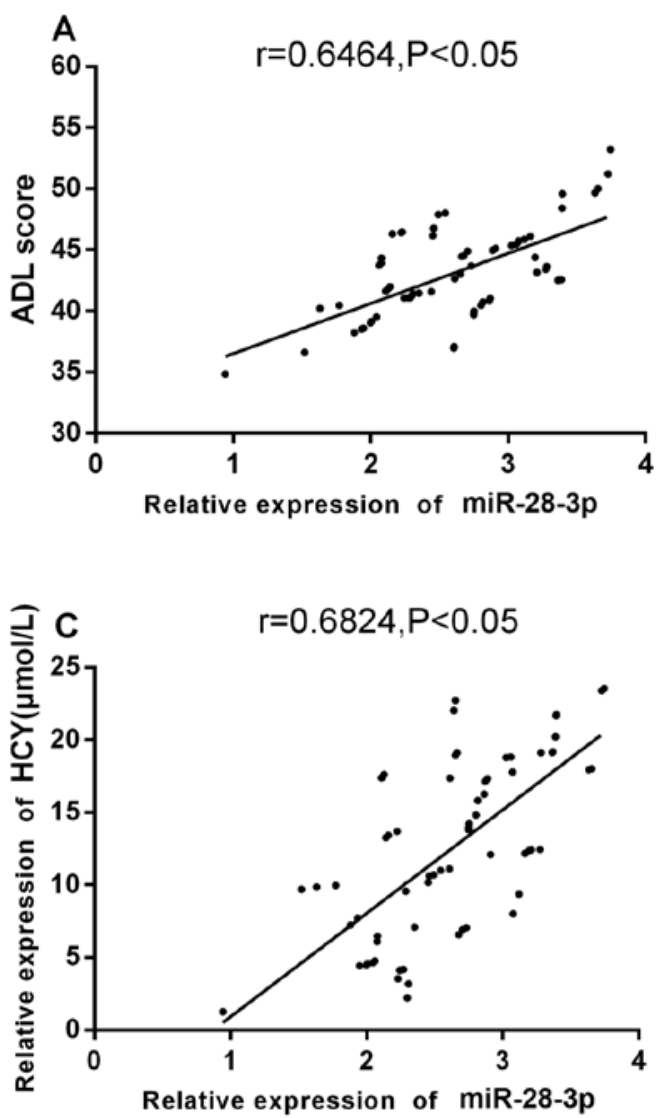
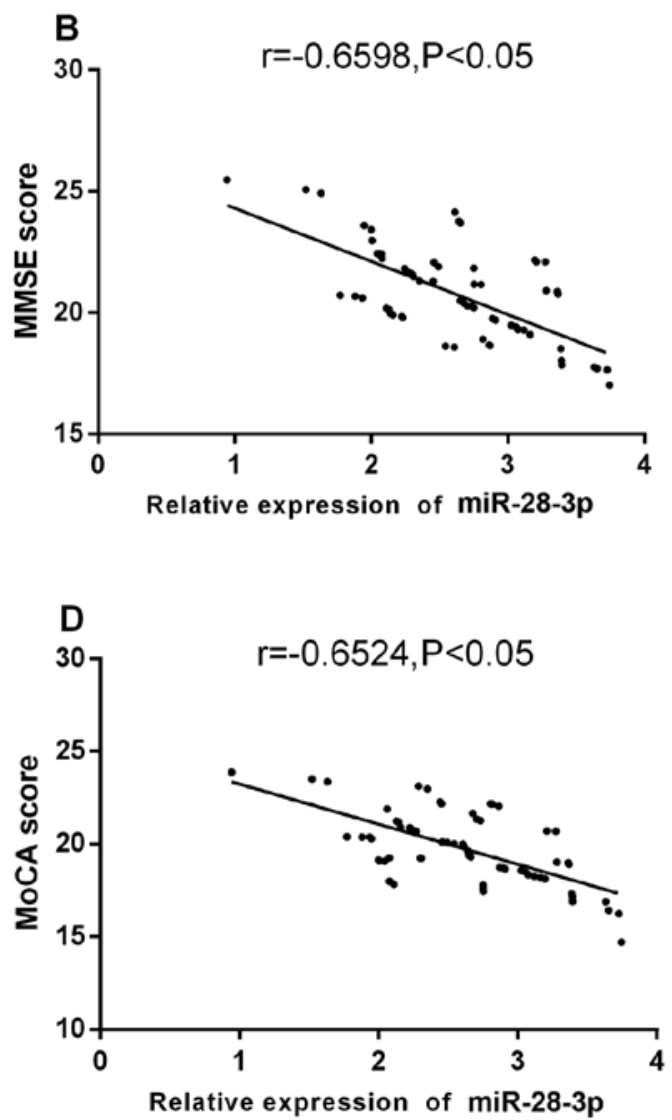

Figure 4. Correlation between serum miR-28-3p level and clinical indexes in AD patients after treatment. Pearson's analysis showing that, (A) after treatment, the serum miR-28-3p level of AD patients was significantly positively correlated with ADL score $(r=0.6464, \mathrm{P}<0.05)$; $(\mathrm{B})$ miR-28-3p level was significantly negatively correlated with MMSE score $(r=-0.6598, P<0.05)$; $(C)$ after treatment, the serum miR-28-3p level of AD patients was significantly positively correlated with Hcy level $(\mathrm{r}=0.6824, \mathrm{P}<0.05)$; (D) miR-28-3p level was significantly negatively correlated with MoCA score $(\mathrm{r}=-0.6524, \mathrm{P}<0.05)$. AD, Alzheimer's disease; ADL, daily living scale; MoCA, Montreal cognitive assessment scale; Hcy, homocysteine; MMSE, mini mental state examination scale.

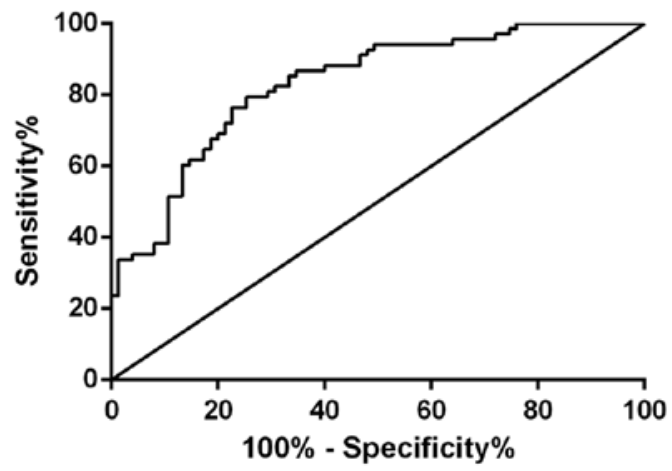

Figure 5. Value of miR-28-3p in diagnosing AD patients. The sensitivity, specificity, AUC and cut-off of miR-28-3p for distinguishing AD patients from normal subjects were $79.41 \%, 74.67 \%, 0.8306(0.7649-0.8963)$, and 2.52 respectively. AD, Alzheimer's disease.

cut-off value of 2.72 for distinguishing $\mathrm{AD}$ patients from $\mathrm{MCI}$ patients.

\section{Discussion}

Pathogenesis of $\mathrm{AD}$ is relatively complex and correlated with inheritance, age, cytoskeleton changes, inflammatory response, nerve transmission obstruction and other factors. Most patients cannot receive timely treatment after suffering from the

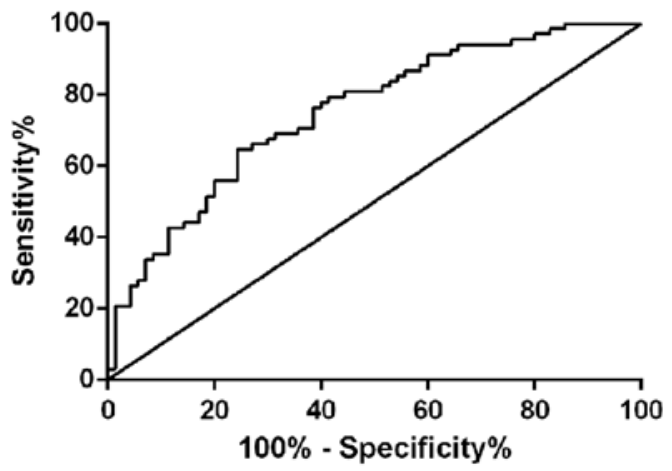

Figure 6. Value of miR-28-3p in diagnosing AD patients. The sensitivity, specificity, AUC and cut-off of miR-28-3p for distinguishing AD patients from MCI patients were $64.71 \%, 75.71 \%, 0.7506$ (0.6704-0.8309), and 2.72 respectively. $\mathrm{AD}$, Alzheimer's disease; $\mathrm{MCI}$, mild cognitive impairment.

illness, which will lead to cognitive impairment of different degrees. The occurrence of the disease will bring economic burden and seriously affect the quality of life of patients (18). The pathological features of AD are mainly vascular amyloidosis, aggregation of abnormally phosphorylated tau protein in cells, formation of neurofibrillary tangles (NFTs), senile plaques formed by deposition of $\beta$-amyloid protein $(A \beta)$, loss of neuronal cells in hippocampus and cerebral cortex $(23,24)$. Donepezil can specifically hinder the degradation of $\mathrm{ACh}$ in the 
brain, reduce the atrophy of brain tissue, and increase the level of choline in the cerebral cortex, thus improving the cognitive function of patients. Donepezil is widely used because of its low hepatotoxicity, long half-life and high safety $(25,26)$.

Recent studies found that there are abundant miRNA in human brain, and about $20-25 \%$ of miRNA are significantly upregulated in AD brain, which is of great significance for identifying and/or diagnosis (27). Most miRNA are highly expressed or specifically expressed in the nervous system, participating in the processes of memory formation, synaptic plasticity and nerve differentiation, and regulating protein synthesis (28-30). Studies have found that miRNA plays an important role in the occurrence and development of AD, and miRNA interference may become a new target for the treatment of AD (31). miRNA has good stability and can be found in plasma and serum samples (32). Jia and Liu (33) detected the expression of miR-223 and miR-519 in serum of AD patients, and found that miR-223 was downregulated and miR-519 was upregulated. miRNA combined diagnosis can be used to predict the occurrence of AD disease. Therefore, detecting the number and types of miRNA in serum samples can effectively reflect the types and severity of the disease, and serum detection is convenient and non-invasive, thus, an ideal early screening method $(34,35)$.

Relevant studies reported that $\mathrm{miR}-28-3 \mathrm{p}$ is a cell limiter, which can inhibit human $\mathrm{T}$ cell leukemia virus, type 1 (HTLV-1) replication and virus infection. miR-28-3p can target a sequence located in the mRNA of viral gag/ pol genomic virus and reduce virus replication and gene expression in transient transgenic cells of HTLV-1 molecular clone (36). miR-28-3p can be detected in various diseases and participates in the occurrence and development of diseases (37-39). Related studies showed that miR-28-5p and miR-28-3p are downregulated in colorectal cancer samples compared with normal colon samples (15). In addition, the differential expression of 27 miRNA between AD patients and normal controls was identified, suggesting that miR-28-3p was significantly upregulated in AD patients (40). This indicated that miR-28-3p is expressed differently in different diseases. The results of this study showed that the miR-28-3p level in normal group was significantly lower than that in AD group and MCI group, while the serum miR-28-3p level in MCI group was significantly lower than that in $\mathrm{AD}$ group $(\mathrm{P}<0.001)$. It is suggested that the cognitive impairment of patients might be related to the upregulation of miR-28-3p expression and the occurrence of $\mathrm{AD}$ was related to the regulation of miRNA. Therefore, combined with previous literature, it was speculated that the upregulation of miR-28-3p in AD might be related to cell survival and cell communication. For example, PI3K-Akt signal transduction, ECM receptor and adhesion spot interaction may be induced and activated by compensatory response to extensive neurodegeneration of AD brain, but more basic experiments are needed to explore the specific action pathway of miR-28-3p (40). The expression of various miRNA in cerebrospinal fluid, the serum of patients with AD and Parkinson's disease, and its relationship with pathological features were detected. It was found that the expression of miRNA was significantly correlated with the severity of the disease, Braak stage, dementia status, plaque and entanglement density, and Louis body (41). However, there is scarce research on the relationship between miR-28-3p and pathological characteristics of AD patients. The present study found that the level of miR-28-3p had no significant correlation with sex, age, smoking or drinking, and the level of miR-28-3p was significant different in different disease courses and severity grades $(\mathrm{P}<0.05)$. It was suggested that the level of miR-28-3p increased gradually with the increase of course and severity, which was significantly correlated with the severity of the disease. Wang et al (42) suggested that miR-206-3p participated in the anti-dementia effect of donepezil and it might be a new pharmacological target for the treatment of AD. Rosignolo et al (43) showed that the level of miR-28-3p in patients with papillary thyroid cancer before operation was significantly higher than that in postoperative and healthy control groups. This study found that the serum miR-28-3p level of AD patients after treatment was significantly lower than that before treatment. This indicated that the combination of donepezil and basic therapy could significantly reduce the miR-28-3p level.

Hcy is produced by metabolism of methionine, which will produce oxidative stress, lead to cell damage and blood-brain barrier damage, and increase the level of brain A $\beta$. Many studies have shown that the increase of Hcy level in peripheral blood of $\mathrm{AD}$ patients was considered to be a risk factor for the occurrence of AD $(44,45)$. Research by $\mathrm{Gu}$ et al (46) showed that Dihuang Yizhi recipe combined with donepezil hydrochloride could significantly improve ADL score, MMSE score and MoCA score of dementia patients with Parkinson's disease. Moreover, it was reported that donepezil could significantly reduce the serum Hcy level of AD patients and had good therapeutic effect (47). Our study found that ADL score and Hcy level of AD patients after treatment were significantly lower than those before treatment, and MMSE score and MoCA score after treatment were significantly higher than those before treatment $(\mathrm{P}<0.05)$. It was suggested that Hcy might be involved in the occurrence of AD, and ADL score, MMSE score and MoCA score could effectively reflect the therapeutic effect. Related studies found that serum miR-223 in AD patients was significantly positively correlated with MMSE score, while serum miR-519 was not significantly positively correlated with MMSE score (33). Correlation analysis showed that serum miR-132 level was negatively correlated with Hcy level, and serum miR-132 level was positively correlated with atrial natriuretic peptide (ANP) level and MMSE score (18). It was presumed that miR-28-3p had the same effect. After analysis, it was found that serum miR-28-3p level in AD patients was significantly positively correlated with ADL score and Hcy level, while miR-28-3p level was significantly negatively correlated with MMSE score and MoCA score. The results showed that with the increase of mental state and quality of life score and the decrease of serum Hcy level, the level of serum miR-28-3p decreased gradually, which effectively reflected the severity of cognitive impairment in AD patients and $\mathrm{miR}-28-3 \mathrm{p}$ level could be used as a therapeutic evaluation of AD disease. Reports showed that the AUC of miR-28-3p was 0.792 (95\% confidence interval: $0.689-0.896)$, and the high expression of miR-28-3p in plasma could be used as a non-invasive and stable biomarker for detecting pulmonary embolism (48). Yu et al (49) used qRT-PCR and then analyzed 
the operating characteristics of the subjects, and found that the expression of miR-28-3p, miR-143-3p, miR-151a-3p and miR-148a-3p were closely related to Helicobacter pylori infection. The above four plasma miRNA groups were expected to be used as non-invasive biomarkers for Helicobacter pylori infection. The present study found that the detection of serum miR-28-3p level could be used for early diagnosis of AD patients, but due to the small sample size and few related studies, a large number of studies are needed to further prove the research results.

This study provided references for the early diagnosis and treatment of AD patients by detecting the level of miR-28-3p in the serum of AD patients. However, no long-term follow-up of $\mathrm{AD}$ patients were carried out to observe their prognosis and whether the expression of miR-28-3p could be used as a prediction of long-term curative effect. Thus, the exact mechanism of miR-28-3p in the occurrence and development of AD needs further research.

In conclusion, donepezil therapy may reduce miR-28-3p level to improve the symptoms of AD patients, and miR-28-3p level can be used as early diagnosis and prognosis evaluation of $\mathrm{AD}$ patients.

\section{Acknowledgements}

Not applicable.

\section{Funding}

No funding was received.

\section{Availability of data and materials}

The datasets used and/or analyzed during the present study are available from the corresponding author on reasonable request.

\section{Authors' contributions}

$\mathrm{XZ}$ wrote the manuscript, interpreted and analyzed the patient data. SW designed the study and performed the experiments. WS was responsible for the analysis and discussion of the data. All authors read and approved the final version of the manuscript.

\section{Ethics approval and consent to participate}

The study was approved by the Ethics Committee of The People's Hospital of Shouguang (Weifang, China). Patients who participated in this research had complete clinical data. Signed informed consents were obtained from the patients and/or guardians.

\section{Patient consent for publication}

Not applicable.

\section{Competing interests}

The authors declare that they have no competing interests.

\section{References}

1. Hampel H, Mesulam MM, Cuello AC, Farlow MR, Giacobini E, Grossberg GT, Khachaturian AS, Vergallo A, Cavedo E, Snyder PJ, et al: The cholinergic system in the pathophysiology and treatment of Alzheimer's disease. Brain 141: 1917-1933, 2018.

2. Kelly SC, He B, Perez SE, Ginsberg SD, Mufson EJ and Counts SE: Locus coeruleus cellular and molecular pathology during the progression of Alzheimer's disease. Acta Neuropathol Commun 5: 8, 2017.

3. Reitz C and Mayeux R: Alzheimer disease: Epidemiology, diagnostic criteria, risk factors and biomarkers. Biochem Pharmacol 88: 640-651, 2014.

4. Alzheimer's Disease International: World Alzheimer Report. Alzheimer's Disease International, London, 2015.

5. Vijayan M and Reddy PH: Stroke, vascular dementia, and Alzheimer's disease: Molecular links. J Alzheimers Dis 54: 427-443, 2016.

6. Morelli A, Sarchielli E, Guarnieri G, Coppi E, Pantano D, Comeglio P, Nardiello P, Pugliese AM, Ballerini L, Matucci R, et al: Young human cholinergic neurons respond to physiological regulators and improve cognitive symptoms in an animal model of Alzheimer's disease. Front Cell Neurosci 11: 339, 2017.

7. Kim SH, Kandiah N, Hsu JL, Suthisisang C, Udommongkol C and Dash A: Beyond symptomatic effects: Potential of donepezil as a neuroprotective agent and disease modifier in Alzheimer's disease. Br J Pharmacol 174: 4224-4232, 2017.

8. Fagan AM, Xiong C, Jasielec MS, Bateman RJ, Goate AM, Benzinger TL, Ghetti B, Martins RN, Masters CL, Mayeux R, et al: Dominantly Inherited Alzheimer Network. Longitudinal change in CSF biomarkers in autosomal-dominant Alzheimer's disease. Sci Transl Med 6: 226, 2014.

9. Jack CR Jr and Holtzman DM: Biomarker modeling of Alzheimer's disease. Neuron 80: 1347-1358, 2013.

10. Absalon S, Kochanek DM, Raghavan V and Krichevsky AM: MiR-26b, upregulated in Alzheimer's disease, activates cell cycle entry, tau-phosphorylation, and apoptosis in postmitotic neurons. J Neurosci 33: 14645-14659, 2013.

11. Hébert SS, Wang WX, Zhu Q and Nelson PT: A study of small RNAs from cerebral neocortex of pathology-verified Alzheimer's disease, dementia with lewy bodies, hippocampal sclerosis, frontotemporal lobar dementia, and non-demented human controls. J Alzheimers Dis 35: 335-348, 2013.

12. Lugli G, Cohen AM, Bennett DA, Shah RC, Fields CJ, Hernandez AG and Smalheiser NR: Plasma exosomal miRNAs in persons with and without Alzheimer disease: altered expression and prospects for biomarkers. PLoS One 10: e0139233, 2015.

13. Kumar P, Dezso Z, MacKenzie C, Oestreicher J, Agoulnik S, Byrne M, Bernier F, Yanagimachi M, Aoshima K and Oda Y: Circulating miRNA biomarkers for Alzheimer's disease. PLoS One 8: e69807, 2013.

14. Long JM, Ray B and Lahiri DK: MicroRNA-153 physiologically inhibits expression of amyloid- $\beta$ precursor protein in cultured human fetal brain cells and is dysregulated in a subset of Alzheimer disease patients. J Biol Chem 287: 31298-31310, 2012.

15. Almeida MI, Nicoloso MS, Zeng L, Ivan C, Spizzo R, Gafà R, Xiao L, Zhang X, Vannini I, Fanini F, et al: Strand-specific miR-28-5p and miR-28-3p have distinct effects in colorectal cancer cells. Gastroenterology 142: 886-896.e9, 2012.

16. Lv Y, Yang H, Ma X and Wu G: Strand-specific miR-28-3p and miR-28-5p have differential effects on nasopharyngeal cancer cells proliferation, apoptosis, migration and invasion. Cancer Cell Int 19: 187, 2019.

17. Hong H, Li Y and Su B: Identification of circulating miR-125b as a potential biomarker of Alzheimer's disease in APP/PS1 transgenic mouse. J Alzheimers Dis 59: 1449-1458, 2017.

18. Paltsev MA, Zuev VA, Kozhevnikova EO, Linkova NS Kvetnaia TV, Polyakova VO and Kvetnoy IM: Molecular markers of Alzheimer disease early diagnostic: Investigation perspectives of peripheral tissues. Adv Gerontol 30: 809-817, 2017 (In Russian).

19. Ma Y, Zhang Z, Chen R, Shi R, Zeng P, Chen R, Leng Y and Chen AF: NRP1 regulates HMGB1 in vascular endothelial cells under high homocysteine condition. Am J Physiol Heart Circ Physiol 316: H1039-H1046, 2019.

20. Matías-Guiu JA, Valles-Salgado M, Rognoni T, Hamre-Gil F, Moreno-Ramos T and Matías-Guiu J: Comparative diagnostic accuracy of the ACE-III, MIS, MMSE, MoCA, and RUDAS for screening of Alzheimer disease. Dement Geriatr Cogn Disord 43: 237-246, 2017. 
21. Zhou G, Liu S, Yu X, Zhao X, Ma L and Shan P: High prevalence of sleep disorders and behavioral and psychological symptoms of dementia in late-onset Alzheimer disease: A study in Eastern China. Medicine (Baltimore) 98: e18405, 2019.

22. Milani P, Vincent Rajkumar S, Merlini G, Kumar S, Gertz MA, Palladini G, Lacy MQ, Buadi FK, Hayman SR, Leung N, et al: $\mathrm{N}$-terminal fragment of the type-B natriuretic peptide (NT-proBNP) contributes to a simple new frailty score in patients with newly diagnosed multiple myeloma. Am J Hematol 91: 1129-1134, 2016.

23. Cacabelos R, Torrellas C and López-Muñoz F: Epigenomics of Alzheimer's disease. J Exp Clin Med 6: 75-82, 2014.

24. Long JM, Maloney B, Rogers JT and Lahiri DK: Novel upregulation of amyloid- $\beta$ precursor protein (APP) by microRNA-346 via targeting of APP mRNA 5'-untranslated region: Implications in Alzheimer's disease. Mol Psychiatry 24: 345-363, 2019.

25. Cavedo E, Grothe MJ, Colliot O, Lista S, Chupin M, Dormont D, Houot M, Lehéricy S, Teipel S, Dubois B, et al; Hippocampus Study Group: Reduced basal forebrain atrophy progression in a randomized Donepezil trial in prodromal Alzheimer's disease. Sci Rep 7: 11706, 2017.

26. Li Q, He S, Chen Y, Feng F, Qu W and Sun H: Donepezil-based multi-functional cholinesterase inhibitors for treatment of Alzheimer's disease. Eur J Med Chem 158: 463-477, 2018.

27. Zhao Y, Pogue AI and Lukiw WJ: MicroRNA (miRNA) signaling in the human CNS in sporadic Alzheimer's disease (AD) - novel and unique pathological features. Int J Mol Sci 16: 30105-30116, 2015.

28. Hohjoh $\mathrm{H}$ and Fukushima T: Expression profile analysis of microRNA (miRNA) in mouse central nervous system using a new miRNA detection system that examines hybridization signals at every step of washing. Gene 391: 39-44, 2007.

29. Schratt GM, Tuebing F, Nigh EA, Kane CG, Sabatini ME, Kiebler $M$ and Greenberg ME: A brain-specific microRNA regulates dendritic spine development. Nature 439: 283-289, 2006.

30. Luo L: Actin cytoskeleton regulation in neuronal morphogenesis and structural plasticity. Annu Rev Cell Dev Biol 18: 601-635, 2002.

31. Satoh J: MicroRNAs and their therapeutic potential for human diseases: Aberrant microRNA expression in Alzheimer's disease brains. J Pharmacol Sci 114: 269-275, 2010.

32. Blondal T, Jensby Nielsen S, Baker A, Andreasen D, Mouritzen P, Wrang Teilum $M$ and Dahlsveen IK: Assessing sample and miRNA profile quality in serum and plasma or other biofluids. Methods 59: S1-S6, 2013.

33. Jia LH and Liu YN: Downregulated serum miR-223 serves as biomarker in Alzheimer's disease. Cell Biochem Funct 34 233-237, 2016

34. Brase JC, Wuttig D, Kuner R and Sültmann H: Serum microRNAs as non-invasive biomarkers for cancer. Mol Cancer 9: 306, 2010.

35. Chen X, Ba Y, Ma L, Cai X, Yin Y, Wang K, Guo J, Zhang Y, Chen J, Guo X, et al: Characterization of microRNAs in serum: A novel class of biomarkers for diagnosis of cancer and other diseases. Cell Res 18: 997-1006, 2008.

36. Bai XT and Nicot C: miR-28-3p is a cellular restriction factor that inhibits human T cell leukemia virus, type 1 (HTLV-1) replication and virus infection. J Biol Chem 290: 5381-5390, 2015.
37. Pospisilova S, Pazourkova E, Horinek A, Brisuda A, Svobodova I, Soukup V, Hrbacek J, Capoun O, Hanus T, Mares J, et al: MicroRNAs in urine supernatant as potential non-invasive markers for bladder cancer detection. Neoplasma 63: 799-808, 2016.

38. Argyropoulos C, Wang K, Bernardo J, Ellis D, Orchard T, Galas D and Johnson JP: Urinary microRNA profiling predicts the development of microalbumin uria in patients with type 1 diabetes. J Clin Med 4: 1498-1517, 2015.

39. Prats-Puig A, Ortega FJ, Mercader JM, Moreno-Navarrete JM, Moreno M, Bonet N, Ricart W, López-Bermejo A and Fernández-Real JM: Changes in circulating microRNAs are associated with childhood obesity. J Clin Endocrinol Metab 98: E1655-E1660, 2013.

40. Satoh J, Kino Y and Niida S: MicroRNA-Seq data analysis pipeline to identify blood biomarkers for Alzheimer's disease from public data. Biomark Insights 10: 21-31, 2015.

41. Burgos K, Malenica I, Metpally R, Courtright A, Rakela B, Beach T, Shill H, Adler C, Sabbagh M, Villa S, et al: Profiles of extracellular miRNA in cerebrospinal fluid and serum from patients with Alzheimer's and Parkinson's diseases correlate with disease status and features of pathology. PLoS One 9: e94839, 2014.

42. Wang CN, Wang YJ, Wang H, Song L, Chen Y, Wang JL, Ye Y and Jiang B: The anti-dementia effects of donepezil involve miR-206-3p in the hippocampus and cortex. Biol Pharm Bull 40: 465-472, 2017.

43. Rosignolo F, Sponziello M, Giacomelli L, Russo D, Pecce V, Biffoni M, Bellantone R, Lombardi CP, Lamartina L, Grani G, et al: Identification of thyroid-associated serum microRNA profiles and their potential use in thyroid cancer follow-up. J Endocr Soc 1: 3-13, 2017.

44. Kamath AF, Chauhan AK, Kisucka J, Dole VS, Loscalzo J, Handy DE and Wagner DD: Elevated levels of homocysteine compromise blood-brain barrier integrity in mice. Blood 107: 591-593, 2006

45. Minagawa $\mathrm{H}$, Watanabe A, Akatsu H, Adachi K, Ohtsuka C, Terayama Y, Hosono T, Takahashi S, Wakita H, Jung CG, et al: Homocysteine, another risk factor for Alzheimer disease, impairs apolipoprotein E3 function. J Biol Chem 285: 38382-38388, 2010

46. Gu C, Shen T, An H, Yuan C, Zhou J, Ye Q, Liu T, Wang X and Zhang T: Combined therapy of Di-Huang-Yi-Zhi with Donepezil in patients with Parkinson's disease dementia. Neurosci Lett 606: 13-17, 2015.

47. Liu X, Zhang J, Xia M, Liu J and Jiang S: Effect of donepezil on Hcy level in serum of Alzheimer's disease patients and correlation analysis of Hcy and dyssomnia. Exp Ther Med 17: 1395-1399, 2019.

48. Zhou X, Wen W, Shan X, Qian J, Li H, Jiang T, Wang W, Cheng W, Wang F, Qi L, et al: MiR-28-3p as a potential plasma marker in diagnosis of pulmonary embolism. Thromb Res 138: 91-95, 2016.

49. Yu J,Xu Q,Zhang X and Zhu M: Circulating microRNA signatures serve as potential diagnostic biomarkers for Helicobacter pylori infection. J Cell Biochem 120: 1735-1741, 2018.

This work is licensed under a Creative Commons

Attribution-NonCommercial-NoDerivatives 4.0 International (CC BY-NC-ND 4.0) License. 